\title{
Neonatal transport in California: findings from a qualitative investigation
}

\author{
Vishnu Priya Akula ${ }^{1} \cdot$ Laura C. Hedli $^{1} \cdot$ Krisa Van Meurs ${ }^{1}$ Jeffrey B. Gould ${ }^{1,2,3} \cdot$ Kan Peiyi ${ }^{3} \cdot$ Henry C. Lee $\mathbb{C}^{1,3}$
}

Received: 2 January 2019 / Revised: 28 April 2019 / Accepted: 17 May 2019 / Published online: 3 July 2019

(c) The Author(s), under exclusive licence to Springer Nature America, Inc. 2019

\begin{abstract}
Objective To identify characteristics of neonatal transport in California and which factors influence team performance. Study design We led focus group discussions with 19 transport teams operating in California, interviewing 158 neonatal transport team members. Transcripts were analyzed using a thematic analysis approach.

Result The composition of transport teams varied widely. There was strong thematic resonance to suggest that the nature of emergent neonatal transports is unpredictable and poses several significant challenges including staffing, ambulance availability, and administrative support. Teams reported dealing with this unpredictability by engaging in teamwork, gathering experience with staff at referral hospitals, planning for a wide variety of circumstances, specialized training, debriefing after events, and implementing quality improvement strategies.

Conclusion Our findings suggest potential opportunities for improvement in neonatal transport. Future research can explore the cost and benefits of strategies such as dedicated transport services, transfer centers, and telemedicine.
\end{abstract}

\section{Introduction}

A neonatal transport team plays an important role in a regionalized healthcare system [1]. They provide stabilization and facilitate transfer of infants from lower acuity hospitals to higher levels of care. In the United States there are gaps in availability between level III or higher obstetric care and neonatal care, as $18 \%$ of obstetric critical care units were found to have no Neonatal Intensive Care Unit (NICU) [2]. This environment is therefore conducive for postnatal

Supplementary information The online version of this article (https:// doi.org/10.1038/s41372-019-0409-7) contains supplementary material, which is available to authorized users.

Vishnu Priya Akula

akulavishnupriya@gmail.com

1 Division of Neonatal and Developmental Medicine, Department of Pediatrics, Stanford University School of Medicine and Lucile Salter Packard Children's Hospital Stanford, Palo Alto, CA, USA

2 California Perinatal Transport System, Stanford University School of Medicine and Lucile Salter Packard Children's Hospital Stanford, Palo Alto, CA, USA

3 California Perinatal Quality Care Collaborative, Stanford University School of Medicine and Lucile Salter Packard Children's Hospital Stanford, Palo Alto, CA, USA transport of critically ill infants. Furthermore, the distribution of specialized care varies across the United States [3]. Retrospective studies have shown associations between duration of transport and increased neonatal mortality [4]. Provision of neonatal intensive care during transport of newborns from a community hospital to a tertiary center is an important determinant of patient outcomes [5].

In the state of California, approximately 7,000 acute neonatal transports are reported each year by the California Perinatal Transport Systems (CPeTS), an organization that is made up of 100 specialized NICUs and maintains a unique and comprehensive dataset of all the neonatal transports in California. Fifty-one-transport teams care for more than $95 \%$ of all neonatal transports in California [6].

Assessment of key infant transport outcomes may be measured by the change in the clinical condition from the time at which the transport team arrives and assumes care until the infant is admitted to the NICU [5]. The TRIPS (Transport Risk Index of Physiologic Stability) score is a practical, validated, physiology-based transport score, which comprises four empirically weighted items (i.e., temperature, blood pressure, respiratory status, and response to noxious stimuli) [5]. Higher TRIPS score has been shown to be associated with mortality [6]. Gould et al. (2013) adapted the Canadian TRIPS score for a California population to assess quality of transport and identified several 
teams that could benefit from quality improvement initiatives $[6,7]$.

There is a gap in knowledge about what factors of neonatal transport relate to the quality of care provided during transport. Prior literature has described components that lead to effective neonatal transport and the neonatal transport team workforce [1, 8]. Roy et al. (1999) described neonatal transport in nine countries throughout the world, exploring the effect of local factors on outcomes [9]. Still, there is scant qualitative research on neonatal transport. The purpose of this study was to examine the perspectives of members of neonatal transport teams in California to identify and explore important characteristics of neonatal transport. Knowing more about what factors influence team performance may inform future quality improvement activities with the goal of improving clinical outcomes.

\section{Methods design}

This qualitative descriptive study thematically analyzed focus group discussions with 19 different transport teams throughout the state of California. In order to select transport teams, we first assessed team performance by analyzing TRIPS scores based on California standards set forth by Gould et al. (2013) [6]. The study was approved by the institutional review board at Stanford University. Informed consent was obtained from all team members who participated in the focus group discussions.

\section{Procedures for participant selection}

We performed a cross-sectional analysis of CPeTS database, which provides administrative and clinical data on every transport (Appendix 1). These data are linked to the datasets from California Perinatal Quality Care Collaborative (CPQCC); CPQCC collects information from admission/discharge forms for all eligible infants cared for at all CPQCC member hospitals representing $>90 \%$ of California NICUs based on definitions developed by the Vermont Oxford Network (VON) [10].

Teams were assessed based on transport data from 2009 to 2012 because we had complete clinical data for that time period. We examined infants that had a record of transport and valid TRIPS score in the CPQCC database. Infants were divided into three diagnostic groups that oftenrequired communication around care coordination and/or active management during transport. Group A had 2075 infants that weighed less than $1500 \mathrm{~g}$ and required positive pressure respiratory support during transport; Group B had 777 infants that required vasopressors at the time of referral or during transport; Group C had 4270 infants that had a major congenital anomaly. The groups were not mutually exclusive and there was some overlap between groups. We chose these categories as broad representations of patient groups for which changes in clinical status during the course of transport may be related to the quality of care.

For each diagnostic group, TRIPS scores were calculated at the time of referral, at the beginning of transport when the team assessed the infant at the referral hospital, and at the end of transport when the team arrived at the receiving hospital. Each TRIPS score was adjusted for birth weight, sex, race, and delivery mode. Multivariable regression models were created. At the beginning of transport, mean TRIPS score for Group A (infants with weight $<1500 \mathrm{~g}$ with respiratory support) was $22 \pm 12$, for Group B (infants on vasopressors) was $32 \pm 10$, and for Groups $\mathrm{C}$ (infants with congenital anomalies) was $7 \pm 12$.

Clinical improvement or deterioration represent two sides of a categorical variable. Clinical improvement was defined by a higher TRIPS score at the beginning of transport compared to the TRIPS score at the end of transport. Clinical deterioration was defined by a lower TRIPS score at the beginning of transport compared to the TRIPS score at the end of transport. Risk adjusted rates of $\%$ clinical deterioration by transport team were obtained. The change in TRIPS score from the beginning of transport to the end of transport is shown in Fig. 1. Transport teams were categorized as high performers or low performers based on the improvement or deterioration of adjusted TRIPS respectively.

Initially, the top 10 high performers and bottom 10 low performers were contacted by email or telephone for a focus group discussion. If a team declined or did not respond, the next team on the list was contacted. Transport teams performing less than 10 transports during the 2009-2012 time period were excluded.

\section{Goal of qualitative analysis}

Our goal in qualitative analysis was not necessarily to distinguish factors between high and low performers; rather, we sought to gain the perspectives of members of transport teams across the performance continuum. Notably, coders (V.P.A. and L.H.) who were blinded to team performance did not notice any appreciable difference between transcripts and could not identify high versus low performers.

\section{Participants and settings}

Of 51 transport teams operating in California, 19 were selected to participate in focus group discussions based on their performance. Nine were high performers and 10 were low performers based on TRIPS scores. Four teams did not respond to our request to interview, and one team agreed but was unable to schedule an interview. The median annual 
Fig. 1 The change in TRIPS score from the beginning transport to the end of transport for all three groups

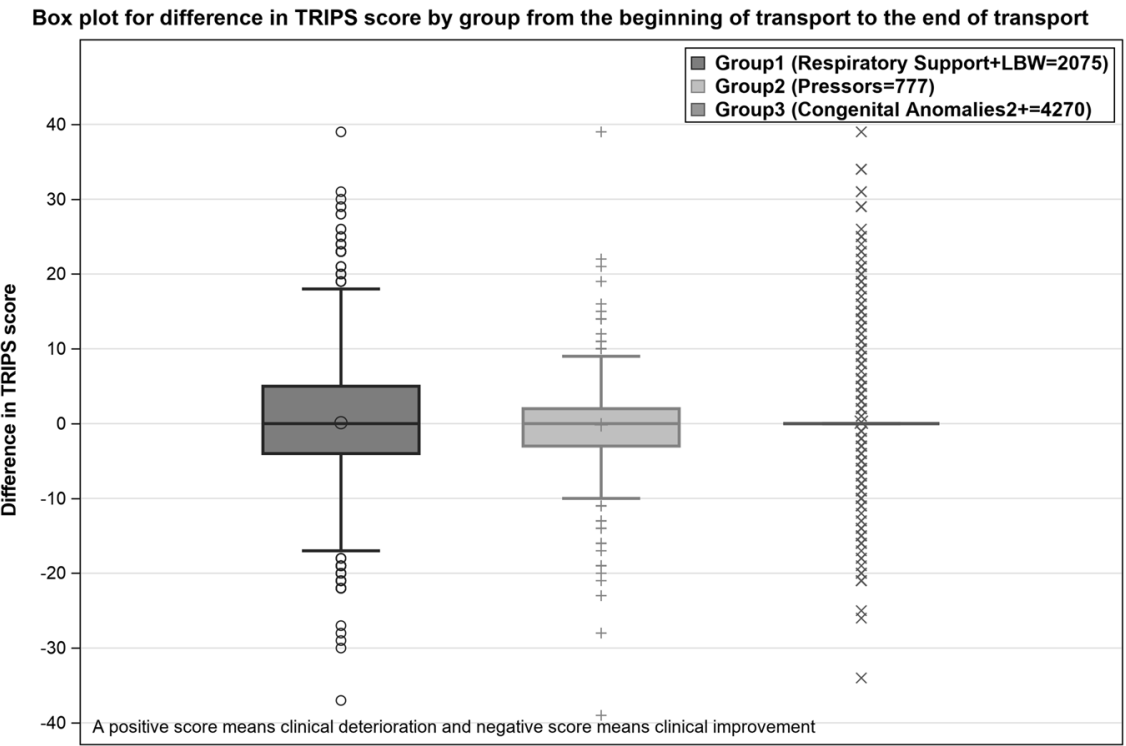

Table 1 Transport team members interviewed

\begin{tabular}{lc}
\hline Title & $N(\%)$ \\
\hline Registered nurses (RNs) & $86(54)$ \\
Neonatologist (MD) & $18(11)$ \\
NICU transport managers & $8(5)$ \\
Emergency medical technicians (EMTs) & $2(1)$ \\
Respiratory care practitioners (RCPs) & $14(9)$ \\
Respiratory therapists (RTs) & $27(17)$ \\
Neonatal nurse practitioners (NNPs) & $3(2)$ \\
Total & $158(100)$ \\
\hline
\end{tabular}

transports performed by participating teams was 57 (interquartile range 44,140) in the period of 2014-2017, which is when we performed the focus group discussions.

Our sample size of 19 focus group units included a total of 158 transport team members (Table 1). Each focus group consisted of members of a single transport team. The average number of people in a discussion was 8-9. Interviews were undertaken with individuals who were in roles that would typically go out on transports for that team. The setting for these discussions was in a conference room type setting in the transport team's hospital. The focus groups were convened between 2015 and 2016.

\section{Procedures for qualitative investigation}

Qualitative investigation was conducted by a single study investigator (V.P.A.) who was blinded to the team performance status. Transport teams were also blinded to their performance status. A semi-structured interview guide (Appendix 2) was prepared and used for all focus group discussions. Because we sought to interview the maximum number of team members, every effort was made to target interviews so that they happened on days when the transport team regularly met or skills days. We developed a twopage, semi-structured interview guide. While we conducted a literature search and found no similar studies to validate our guide, our guide was used only as a starting point for our focus group discussions. The facilitator allowed transport team members to direct the discussion toward points they felt were most relevant. All discussions were recorded with the permission of the transport team members. The recordings were then professionally transcribed.

\section{Qualitative analysis}

Two investigators (P.A. and L.H.), both masked to team performance status and working independently, closely read transcripts and developed codes using qualitative analytic methods adapted from Braun and Clarke (2006) [11]. Braun and Clark propose thematic analysis as an accessible approach to analyzing qualitative data that is theoretically flexible [11]. We analyzed our study transcripts thematically, which allowed themes to emerge within and across transcripts in a way that was highly representative of the data. Through discussions that established consensus, investigators consolidated codes under each theme into central organizing concepts and identified relationships between concepts. Illustrative quotes from the transcripts were grouped under each concept. We used consensus among investigators (V.P.A., L.H., and H.L.) to solidify relationships between concepts and establish which quotes and data elements to highlight in the manuscript. Quotes were edited minimally for readability. 


\section{Results}

The process of transport was described by each of the 19 neonatal transport teams. Broadly speaking, the same general series of events occur to initiate transport: (1) the referral hospital places a call, (2) an intake form is used to collect information about the baby, and (3) the transport team prepares to leave for the referral hospital. Overall, teams reported that multiple phone calls often occurred between referral and receiving hospital personnel to check bed availability, staffing, and insurance, and to gather clinical information. Of note, three teams used the services of a transfer center with a centralized number at the receiving hospital. A transfer center streamlined the transport process by connecting the accepting physician and nurse to the referring physician and nurse to obtain pertinent information; the center often documented this exchange on the intake form. The transfer center also coordinated the logistics of transport-ambulance, helicopter, or fixed wing - while the team prepared to leave. One team member said the transfer center they use helps us to have adequate information and to be prepared to make the transport go as quickly as possible.

Team composition varied between teams and was based on a variety of factors including acuity of transport, the gestational age and/or respiratory status of the baby, and the skill level and experience of team members. Some teams had a set composition no matter the clinical situation at the referral hospital. Several teams that managed higher acuity cases required that a physician or transport specialist be part of the transport team; others required a second registered nurse for critical cases. Some teams operated without a physician on transport.

Transport modes included ground, helicopter, and fixed wing. Ambulance was the most common mode of transport, with 11 of 19 teams using this mode only. Seven teams used all three modes of transport, and one team used ambulance and fixed wing. The majority of hospitals transported infants to higher levels of care; only two hospitals reported transports for insurance reasons. For emergent transports, most teams reported having a goal of departing for the referral hospital 30-60 min following the initial referral call. All transport teams were able to reach the attending neonatologist at the receiving hospital by cellphone at almost any time during transit. One team specifically reported using live video in the ambulance during transport to show the baby to a neonatologist at the receiving hospital.

\section{What makes transport unpredictable?}

While a transport team can plan ahead to manage a scheduled transport, all teams emphasized the unpredictable nature characterizing emergent neonatal transport. The most important variable is timing there is no way of knowing when a request for emergent transport will be received (e.g., day vs. night, weekday vs. weekend, holiday). Duration of transport varies based on traffic, weather, and interfacility distance. Many teams commented on how the clinical situation may change between the time a call comes in from a referral hospital and the time the transport team arrives at the referral hospital, making it difficult for a transport team to adequately prepare to manage certain patients. Transport teams reported working with a wide range of referral hospitals, from an emergency room at a community hospital up to a level III NICU. Accordingly, teams reported variation in the skill levels of staff at referral hospitals.

\section{With unpredictability comes challenges}

The unpredictability of transport may contribute to variable times for departure from the receiving facility based on staffing availability, ambulance availability, and ambulance parking location. If the baby is sicker and requires a helicopter or fixed wing transport, air transport restricts the operation of the transport team and their communication with the neonatologist at the receiving hospital. Because revenue streams for transport are variable, teams cited a lack of support from hospital administration.

\section{Staffing}

Staffing was cited as a major reason to decline transport. The majority of teams were staffed from personnel in-house at the receiving hospital or called in from home. While teams reported more flexibility in staffing during the day than at night, the uncertainty in the timing of the request for transport often led to difficulties in staffing for registered nurses (RNs) and respiratory therapists (RTs), who work set 8 or 12 -h shifts.

One of the biggest problems we always find is getting the correct staff together. When you realize a transport is going to take somewhere between 3-5 h, and if shifts are ending, shifts are starting, it is sometimes hard to get someone to agree to stay over for an extra couple of hours after their shift has ended.

Both RNs and RTs were frequently pulled from other assignments or delivery room coverage, and this either delayed times to departure for transport teams and/or left gaps in coverage for inpatient care. RNs and RTs were reported to have different shift timing, creating two separate coverage issues. Teams cited several challenges specific to obtaining an RT for transport: RT staffing on transport teams was at times voluntary; RTs could have different expectations for response time for a transport call compared 
to RNs; and RTs could be pulled from an adult care floor without adequate training to manage a NICU patient.

To solve staffing problems, several teams expressed that having a dedicated transport team would be ideal.

Just having a transport team readily available is our biggest issue and obstacle in improving our entire process. We currently do not have the budget, or the resources and staffing, to continuously have a transport team on call, $24 \times 7$.

\section{Ambulance}

Quickly obtaining an ambulance that could accommodate the needs of a neonate was challenging given the unpredictability in the timing of transport and traffic. Very few teams had a dedicated, custom-fit rig to transport a NICU patient available at all times and parked close to the hospital; however, those that did reported being able to mobilize quickly.

Teams reported miscommunications when placing a request for pick-up with the ambulance company and drivers who were not readily available. Some rigs did not have space to accommodate all members of the team, equipment was not always strapped down, and seatbelts restricted team members' range of motion. Teams explained that not all ambulances were outfitted to transport an incubator or have compatible power converters for equipment.

The ambulance that we were receiving did not have inverters, they did not have the vent. We have a pneumatic system, so I needed medical air and oxygen and they were only providing us with oxygen and not the metal gear. I could not use our machines. I had to rely on our tanks.

Of note, many teams were passionate about the poor suspension in ambulances, especially in older rigs and even in newer, dedicated rigs. The suspensions made for a bumpy ride, which they believed could contribute to negative outcomes such as intraventricular hemorrhage. Teams cited ways to protect the baby including using a gel mattress, placing a bean bag on top of the baby, avoiding freeways, and driving slowly.

The suspensions make it very difficult to assess the babies on a continuing basis with accurate information from the monitor because there is so much artifact generated. I think in some cases the rides are painful for the patients, and in other cases they may be downright dangerous and result in very negative outcomes.

\section{Air transport}

The use of helicopter and fixed wing often poses its own set of problems. Issues identified by transport teams included low lighting, loud noise that prevents communication, lack of space that permits only one person to have access to the baby, and limited cell signal that may prevent the team from communicating with the neonatologist at the receiving hospital.

We usually go for the sicker kids in the helicopter and I want to have my ducks much more in a row. I want to have everything nailed down and in place in a helicopter. It is very, very cramped and loud-a difficult place to actually execute, work, and communicate.

\section{Administration}

Neonatal transport is high-risk and often low-volume compared to inpatient care and has an unpredictable revenue stream. Even though a transported patient ultimately brings in revenue to the hospital, transport teams said they received varying support from hospital administrations, with only a few teams reporting feeling adequately supported. Many teams felt a lack of support from the administration with respect to ensuring staff for transport and securing funding for skills days and equipment. One team cited relying on their own fundraising efforts to obtain smaller items.

Transport is of the nature where transport is a necessity for hospitals, but it is not a money maker. The days that the baby is in the bed is what's making the money. From a high-level administrative perspective, they do not see the value because it is not creating revenue. So, it's a constant fight for transport teams all over the nation to prove your wealth, so to speak.

\section{Dealing with the unpredictability of transport}

While the nature of transport is unpredictable and therefore poses several challenges, teams said that their performance is strengthened by their experiences working together and with referral hospitals. Overall, teams reported that preparing for changes in the clinical situation and planning ahead were critical to transport success. Outcomes were improved through training, debriefing, and outreach efforts. 


\section{Teamwork}

When asked what worked well in their current system, many teams talked about teamwork. They described the experiences they had working together contributing to their overall strength and performance. Experienced teams reported gaining the trust of a physician, so they could make critical decisions on transport and later notify the physician. Members described having respect and trust in one another and good verbal and nonverbal communication during transports. One team member said they pretty much know what the other's thinking, and another reported being on the same team is like second nature.

Few hospitals had a dedicated transport team, whereby they assigned RNs, RTs, and even physicians to be on call for transport at all times. Dedicated transport teams may operate differently depending on hospital scheduling and administration. For example, while on call and not on transport, some transport teams are required to attend deliveries, place central lines, or even pick up patient assignments. Overall, those interviewed who were on dedicated transport teams described teams composed of a group of members who have significant experience working together and the opportunity to maintain their skills as a team.

Our team has been together for 15 years. Almost all of us started the team together. When we were new, we did the simplest transports. Over the years of working together, we have developed our knowledge, skills, and our confidence in the types of patients that we are seeing from our facilities. Being able to work with the same team members is a big deal.

\section{Experience with referral hospitals}

A clinical situation may change between when a call comes in from the referral hospital and when the transport team arrives, and some teams reported that information on the intake form was often insufficient or inaccurate. Therefore, teams often relied on prior experience with referral hospitals to assess: (1) whether the information they received on the intake form was correct, and (2) how much they could rely on staff at the referral hospital to help with patient stabilization. Based on prior experience with referral hospitals, teams prepared differently depending on which referral hospital requested transport.

We recognize that with one particular hospital we get a good clear report; they manage babies very well. We have another hospital who does very well also. We have a third hospital that does not manage their sick babies very well. So, we know when we go to that particular hospital, in spite of the report, things might be better or worse. We know that whereas the other two hospitals make every effort to get blood drawn and an IV started along with that, the third hospital may not.

Several teams said they respected the efforts of the staff at referral hospitals. Teams worked to establish good relationships and open communication with referral hospitals, which they said contributed to staff at referral hospitals taking a more active role in helping with care once the transport team arrived. It is critical to be diplomatic.

Our team members, our team leaders, and our medical control physicians have developed a very healthy respect for the referring physician base. We do not show any signs of disrespect for their knowledge base or what they have done.

Teams acknowledged that referral hospitals may not be equipped to deal with high acuity cases (especially in community hospital emergency rooms, for example), and may not have the time or resources to simultaneously stabilize a sick baby and prepare a report for the transport team. Notably, teams reported the burden of providing an up-to-date report was alleviated if the referral and receiving hospitals shared the same electronic medical record system. The transport team was able to receive the most current information on the patient's clinical status and view labs and x-rays en route. A few teams within the same medical group were even able to use videoconferencing to visualize the patient and make clinical recommendations to the referral team prior to the transport team's arrival.

The video visits have actually enhanced the transport or at least the stabilization of the baby immensely. We can look at their ventilator settings and make recommendations, and our $R T$ can check the setup, prongs, and interface.

\section{Planning ahead and preparing for future transports}

Planning Almost all teams stressed that it is necessary to prepare for any situation given the many variables inherent in the transport process. Many teams relied on having transport bags with pre-stocked equipment, common medications, and supplies necessary for a range of clinical scenarios. Supplies were labeled, checked periodically, and had designated locations in the bags.

I have been transporting for 33 years, both PICU and NICU patients, and you never get the right 
information and you never get enough information. I train to prepare for the worst and hope for the best, and so ideally, we should be going out the door with every piece of equipment you would need to anticipate anything.

Training Each transport team had their own individualized requirements for maintaining skills and training new members, and training varied widely between teams. Types of training included ride alongs; skills labs; simulations; delivery room training; drills with the ambulance and helicopter; Sugar Temperature Airway Blood Pressure Lab work \& Emotional support (STABLE) program for RNs; NICU training; Federal Aviation Administration (FAA) training; Morbidity \& Mortality (M\&M) attendance; individual training; and testing on specific skills including intubations, umbilical lines, arterial lines, and needle thoracentesis. Duration of training new members also varied. Typically, training for RNs was more involved than RTs. In one case, RTs were reported to be able to go out on transport with as little as 2 weeks of training, whereas some teams required RNs to have at least 2 years of training.

Debriefing and quality improvement Debriefing allowed teams to assess transport outcomes and also understand the thought processes behind the actions of each team member during transport. Teams debriefed immediately after complex and critical transports to identify what went well and what could be better. One team member commented that debriefing improves our function for our next transport. Some cases were presented at M\&M meetings, neonatal conferences or transport meetings; teams had the chance to discuss cases in order to improve clinical outcomes during subsequent transports.

Issues identified during debriefing sometimes became topics for larger quality improvement initiatives. Different teams used metrics to assess the quality of transport, such as out-the-door time, blood glucose, and temperature. These metrics were reviewed at different times-monthly, quarterly, or yearly. Of note, one team provided data-driven feedback to each member of the team on their individual out-the-door time metrics. This process improved overall out-the-door times.

Outreach Transport teams often engaged in training and outreach efforts with referral hospitals and, more broadly, in the communities they covered. A couple of teams reported sending a physician to the referral hospital before the transport team's arrival; the physician used this time as a teaching opportunity for staff at the referral hospital. Outreach efforts included offering mock codes and trainings on the Neonatal Resuscitation Program, simulation, golden hour, and stabilizations. One team even provided referral hospitals with what they called a "tiny baby box," which had all the supplies required for an extremely low birth weight (ELBW) baby.

Table 2 summarizes the challenges and strengths that may impact outcomes of emergent neonatal transport.

\section{Discussion}

We conducted a qualitative evaluation across a spectrum of neonatal transport teams in the state of California. In total, we gathered the perspectives of 19 different transport teams, with 158 transport team members participating in focus group discussions. A significant finding was that team composition was variable, reflecting differences in team sizes, areas served, and patient acuity. This result is supported by earlier work by Karlsen et al. (2011), who identified 26 different compositions among 335 neonatal transport teams in the U.S [8]. Many teams we interviewed reported that they operated without a neonatologist on transport. The absence of a neonatologist may not represent a deficiency in care as the literature on transports has shown no significant difference in adverse events between nurseled or physician-led transport teams [12-14].

There was strong thematic resonance to suggest that unpredictability is a key feature that impacts team performance during emergent transports. Unpredictability poses several significant challenges to the transport team. Given that most transport teams relied on staff who were in house caring for other patients and/or staff coming in from home, these teams often reported delays in time of departure due to lack of sufficient personnel. Prior investigation has revealed that NICUs in the U.S. are already understaffed for RNs relative to national guidelines [15]. The majority of the teams we interviewed expressed concerns over both RN and RT staffing, a concern echoed by Kornelsen and colleagues (2018) who reported on high acuity rural transport [16]. Hospital-based staffing for transport teams is economical but takes personnel away from in house patient assignments, delays departure, and lacks flexibility. Our findings suggest dedicated transport services, including dedicated transport personnel, may improve expediency and efficiency. In Maryland, one hospital found that the formation of a dedicated NICU staff nurse position, where responsibilities were restricted to going on transports and attending deliveries requiring the NICU team, resulted in improved clinical outcomes and optimized the use of nursing full-time equivalents [17].

From an administrator perspective, it may be difficult to find sufficient workload to justify the costs of maintaining dedicated transport services including dedicated transport personnel and dedicated rigs available onsite. Future 
Table 2 Challenges and strengths that may impact outcomes of emergent neonatal transport

Challenges

$\mathrm{RN}$ and RT staffing

Staffing is a common reason for declining transport and major contributor to delayed departure times. Transport team members are often pulled from inpatient assignments or called in from home. Staff expectations, lack of training, and variable shift times are factors that may affect transport outcomes.

Ambulance If there is no dedicated rig onsite at the receiving hospital, factors including traffic and transport timing affect the ability for a team to quickly obtain ground transportation. Additional problems may include miscommunication with the ambulance company, lack of proper equipment in the rig, and seatbelts that restrict range of motion of team members. Of note, poor ambulance suspensions make for bumpy rides that may contribute to negative outcomes like intraventricular hemorrhage.

Air transport

Helicopter and fixed wing are characterized by low lighting, cramped spaces, and loud noises that restrict communication and access to the baby. The transport team may not be able to communicate with neonatologist at the receiving hospital because of noise and low cell signal in the air.

Administration

While transport brings in patients to the receiving hospital, it is not seen as a moneymaker. Teams feel a lack of support from administrations with respect to ensuring staff for transport and securing funding for skills days and equipment.

Intake form

Standardized transport forms may be dated, insufficient, or inaccurate. To get necessary clinical information, additional phone calls to the referral hospital may be required.

\section{Strengths}

Teamwork

Experience with referral hospitals

Planning

Training

Debriefing \& quality improvement

Outreach

Hierarchy
Team configuration varies, and no one combination is recommended. Established working relationships among transport team members and experience doing transports together contributes to overall strength and performance. Dedicated teams often have significant experience working together and maintaining skills as a team.

Experience with referral hospitals helps with managing expectations for transport. Good, open communication with referral hospitals may lead to staff at referral hospitals taking a more active role. Care coordination between receiving and referral hospitals is improved with access to a shared electronic medical record and/or video conferencing capabilities.

Prepare for anything because what will happen cannot be known. Teams may mentally prepare by prebriefing and physically prepare by pre-stocking transport bags with equipment, common medications, and supplies necessary for a range of clinical scenarios.

Training for transport team members varies widely. Also variable is the duration of training for new members.

Debriefing after transport and case review improve the quality of transports. Quality improvement efforts vary.

Outreach programs based on feedback from referral hospitals help train staff at referral hospitals. These programs may improve relationships and communication between receiving and referral hospitals and patient care.

Transport teams are characterized by some sort of hierarchical leadership. Leadership and support from those in supervisory roles is important to the success of transport. research is needed to measure the fiscal impact of transport services, including mode of transport costs and emergency medical response services. Parts of Europe have developed centralized transfer services with mixed results. A study of Neonatal Emergency Transport Services operating in Liguria, Italy found that teams needed to carry out 200-350 transports per year in order to be financially viable and for staff to acquire and maintain the necessary skills [18]. In the U.S., Karlsen and colleagues (2011) found that $85 \%$ of the unit-based transport teams carried out less than 200 transports each year, whereas $73 \%$ of dedicated transport teams carried out more than 200 transports each year [8]. Our participating teams, most of which were unit-based, carried out a median annual transport volume of 57. More research is needed to calculate the cost of maintaining a dedicated team and the number of transports they need to perform to justify the cost. Several of the teams we interviewed said that the administration felt that neonatal transport was a "money loser" for the hospital, yet no metric exists to measure the revenue brought to the hospital by each patient who is transported.

Our study generates novel insights about the ways transport teams deal with the unpredictable nature of transport. There was strong congruence across transcripts around the following central concepts: teamwork is beneficial, experience with staff at referral hospitals helps manage expectations, and planning for all clinical scenarios is critical. While all teams engaged in training, debriefing and quality improvement, there was wide variation between teams on each of these measures.

Table 3 suggests several areas for growth that may improve the outcomes of emergent neonatal transport based 
Table 3 Recommended areas of growth to improve outcomes of emergent neonatal transports

Recommended areas for growth

Dedicated transport services

Overall, dedicated transport teams are composed of providers who have significant experience working together and the opportunity to maintain their skills as a team. While having a dedicated team may not be feasible for a hospital, restricting in house responsibilities for certain personnel, thereby creating a dedicated transport staff, may improve expediency and efficiency.

Transfer centers $\quad$ Transfer centers streamline communication and ensure all requisite intake information is collected. The use of call centers reduces the number of phone calls between referral and receiving hospitals, thereby helping to expedite the time to departure.

Telemedicine

- Videoconferencing initiated by the referral hospital helps the neonatologist at the receiving hospital and the transport team evaluate and manage the patient remotely.

- Integrated electronic medical record between referral and receiving hospitals ensures free flow of real-time information, saves time, and improves communication and care coordination.

- Use of a smartphone with video capabilities during transport helps the neonatologist visualize the patient while the transport team is en route to the receiving hospital.

- Webcam access may help alleviate parents' anxiety

on our findings. In addition to having dedicated transport services, establishing transfer centers and implementing telemedicine may improve and streamline communication throughout the transport process. Positive outcomes were reported by teams that used a transfer center, and those that were part of a health care system where the transport team, neonatologist, and referral hospital all had access to realtime updates from the electronic medical record. Given that the cost for just the technical equipment at a transfer center is estimated to be about $\$ 89,000$ [14], a free-standing transfer center shared by several hospitals or other units within a hospital could potentially be an economical solution. Similarly, tele-neonatology may facilitate collaboration between the receiving hospital, transport team, and referral hospital $[19,20]$. It has been shown to improve the quality of care provided during high-risk newborn resuscitations and improve assessment of patient stability during transport management of simulated newborns [21-23]. Webcam access to infants has also been shown to alleviate parental anxiety and improve parent-infant bonding [24].

Our study has several limitations. Not all transport team members were present at our focus group discussions, meaning that certain perspectives may not have been captured in our analysis. It is also possible there have been changes in team composition or performance during the period when TRIPS scores were analyzed and the time when focus groups were convened. It was not within the scope of this study to link the perspectives of health care providers to clinical performance outcomes or other significant metrics like out-the-door time. We interviewed transport teams only in California and recognize that the operation of teams in other states in the U.S. and around the world may differ. More research is needed to validate perspectives and determine best practices for neonatal transport.

\section{Conclusion}

This is the first qualitative, descriptive study to address the perspectives of neonatal transport team members in California and highlights the unpredictable characteristics of neonatal transport. Thematic analysis revealed among teams common challenges as well as strengths that help counter the unpredictability inherent in neonatal transport. Our study provides a basis for future work in quality improvement by providing potential points of assessment that may serve as process and structure measures. We recommend future studies to determine the cost-benefit of dedicated transport services, telemedicine, and transfer centers.

Acknowledgements We would like to acknowledge the important contributions of Abhinav Kareddy, University of California, Los Angeles (review of transcripts and illustrative quotes).

Funding We appreciate the support of the Maternal \& Child Health Research Institute, Lucile Packard Foundation for Children's Health and Stanford CTSA (grant number UL1 TR001085).

\section{Compliance with ethical standards}

Conflict of interest The authors declare that they have no conflict of interest.

Publisher's note: Springer Nature remains neutral with regard to jurisdictional claims in published maps and institutional affiliations.

\section{References}

1. Lupton BA, Pendray MR. Regionalized neonatal emergency transport. Semin Neonatol. 2004;9:125-33.

2. Brantley MD, Davis NL, Goodman DA, Callaghan WM, Barfield WD. Perinatal regionalization: a geospatial view of perinatal critical care, United States, 2010-2013. Am J Obstet Gynecol. 2017;216:185 e181-185 e110. 
3. Wang HE, Yealy DM. Distribution of specialized care centers in the United States. Ann Emerg Med. 2012;60:632-7. e637

4. Mori R, Fujimura M, Shiraishi J, Evans B, Corkett M, Negishi H, et al. Duration of inter-facility neonatal transport and neonatal mortality: systematic review and cohort study. Pedia Int. 2007;49: 452-8.

5. Lee SK, Zupancic JA, Pendray M, Thiessen P, Schmidt B, Whyte R, et al. Transport risk index of physiologic stability: a practical system for assessing infant transport care. J Pedia. 2001;139:220-6.

6. Gould JB, Danielsen BH, Bollman L, Hackel A, Murphy B. Estimating the quality of neonatal transport in California. J Perinatol. 2013;33:964-70.

7. Ratnavel N. Evaluating and improving neonatal transport services. Early Hum Dev. 2013;89:851-3.

8. Karlsen KA, Trautman M, Price-Douglas W, Smith S. National survey of neonatal transport teams in the United States. Pediatrics. 2011;128:685-91.

9. Roy N, Langford S. Newborn transport around the world. Semin Fetal Neonatal. 1999;4:219-35.

10. CPQCC. California Perinatal Quality Care Collaborative. 2016. https://www.cpqcc.org/about/our-members. Accessed 2 April 2019.

11. Braun V, Clarke V. Using thematic analysis in psychology. Qual Res Psychol. 2006;3:77-101.

12. Colyer E, Sorensen M, Wiggins S, Struwe L. The effect of team configuration on the incidence of adverse events in pediatric critical care transport. Air Med J. 2018;37:186-98.

13. King BR, King TM, Foster RL, McCans KM. Pediatric and neonatal transport teams with and without a physician: a comparison of outcomes and interventions. Pedia Emerg Care. 2007;23:77-82.

14. Medicine SoT, AAP. Guidelines for air and ground transport of neonatal and pediatric patients. 4th ed. American Academy of Pediatrics Itasca, IL, USA, 2015.
15. Rogowski JA, Staiger D, Patrick T, Horbar J, Kenny M, Lake ET. Nurse staffing and NICU infection rates. JAMA Pedia. 2013;167:444-50.

16. Kornelsen J, Hobbs B, Buhler H, Kaus R, Grant K, Lamont SC, et al. High acuity rural transport: findings from a qualitative investigation. Rural Remote Health. 2018;18:4316.

17. Diehl BC, Frank K. A multifaceted nursing role to comply with neonatal transport regulations in Maryland: The TR/DR Nurse. Adv Neonatal Care. 2016;16:360-8.

18. Bellini C, Pasquarella M, Ramenghi LA, Ambrosino D, Sciomachen AF. Evaluation of neonatal transport in a European country shows that regional provision is not cost-effective or sustainable and needs to be re-organised. Acta Paediatr. 2018; 107:57-62.

19. Cornette L. Contemporary neonatal transport: problems and solutions. Arch Dis Child Fetal Neonatal Ed. 2004;89:F212-214.

20. Beck JA, Jensen JA, Putzier RF, Stubert LA, Stuart KD, Mohammed H, et al. Developing a newborn resuscitation telemedicine program: a comparison of two technologies. Telemed J E Health. 2018;24:481-8.

21. Fang JL, Campbell MS, Weaver AL, Mara KC, Schuning VS, Carey WA, et al. The impact of telemedicine on the quality of newborn resuscitation: a retrospective study. Resuscitation. 2018; 125:48-55.

22. Fang JL, Collura CA, Johnson RV, Asay GF, Carey WA, Derleth DP, et al. Emergency video telemedicine consultation for newborn resuscitations: the Mayo Clinic experience. Mayo Clin Proc. 2016;91:1735-43.

23. Umoren RA, Gray MM, Schooley N, Billimoria Z, Smith KM, Sawyer TL. Effect of video-based telemedicine on transport management of simulated newborns. Air Med J. 2018;37:317-20.

24. Thibeau S, Ricouard D, Gilcrease C. Innovative technology offers virtual visitation for families. J Contin Educ Nurs. 2012;43:439-40. 